Témoigner Témoigner. Entre histoire et mémoire

Getuigen Revue pluridisciplinaire de la Fondation Auschwitz

$121 \mid 2015$

Violences radicales en scène

\title{
Créations artistiques au Rwanda après le génocide des Tutsi
}

Transmettre la mémoire, reconstruire la culture

Ariane Zaytzeff

\section{(2) OpenEdition}

Journals

Édition électronique

URL : https://journals.openedition.org/temoigner/3331

DOI : $10.4000 /$ temoigner.3331

ISSN : 2506-6390

Éditeur :

Éditions du Centre d'études et de documentation Mémoire d'Auschwitz, Éditions Kimé

Édition imprimée

Date de publication : 1 octobre 2015

Pagination : 84-94

ISSN : 2031-4183

Référence électronique

Ariane Zaytzeff, «Créations artistiques au Rwanda après le génocide des Tutsi », Témoigner. Entre histoire et mémoire [En ligne], 121 | 2015, mis en ligne le 01 octobre 2016, consulté le 04 février 2022. URL : http://journals.openedition.org/temoigner/3331; DOI : https://doi.org/10.4000/temoigner.3331 


\section{Créations artistiques au Rwanda après le génocide des Tutsi}

Transmettre la mémoire, reconstruire la culture

$\rightarrow$ Par Ariane Zaytzeff, Tisch School of the Arts New York University

_ Répétition

de Shadows of Memory, mise en scène d'Azeda.

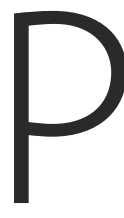

lacée entre le témoignage d'un rescapé et le discours du président de la République du Rwanda, la pièce de Mashirika est partie prenante de la commémoration officielle organisée par le gouvernement et pose la question du rôle des arts vivants dans la performance de la mémoire du génocide des Tutsi au Rwanda. Je choisis ici le terme «performance », qui porte étymologiquement la notion d'exécution ou d'accomplissement, pour rappeler que, comme le souligne Paul Connerton, la mémoire n'est pas une reproduction, mais une construction du passé (Connerton, 1989, 27). Les représentations artistiques qui travaillent avec la mémoire du génocide ne se saisissent donc pas d'un matériau déjà présent, mais contribuent à sa création, et je questionnerai la nature de leurs interventions. En posant la question de ce que peuvent faire les arts vivants face à la violence extrême du génocide, il s'agira de montrer qu'ils peuvent offrir plus qu'une

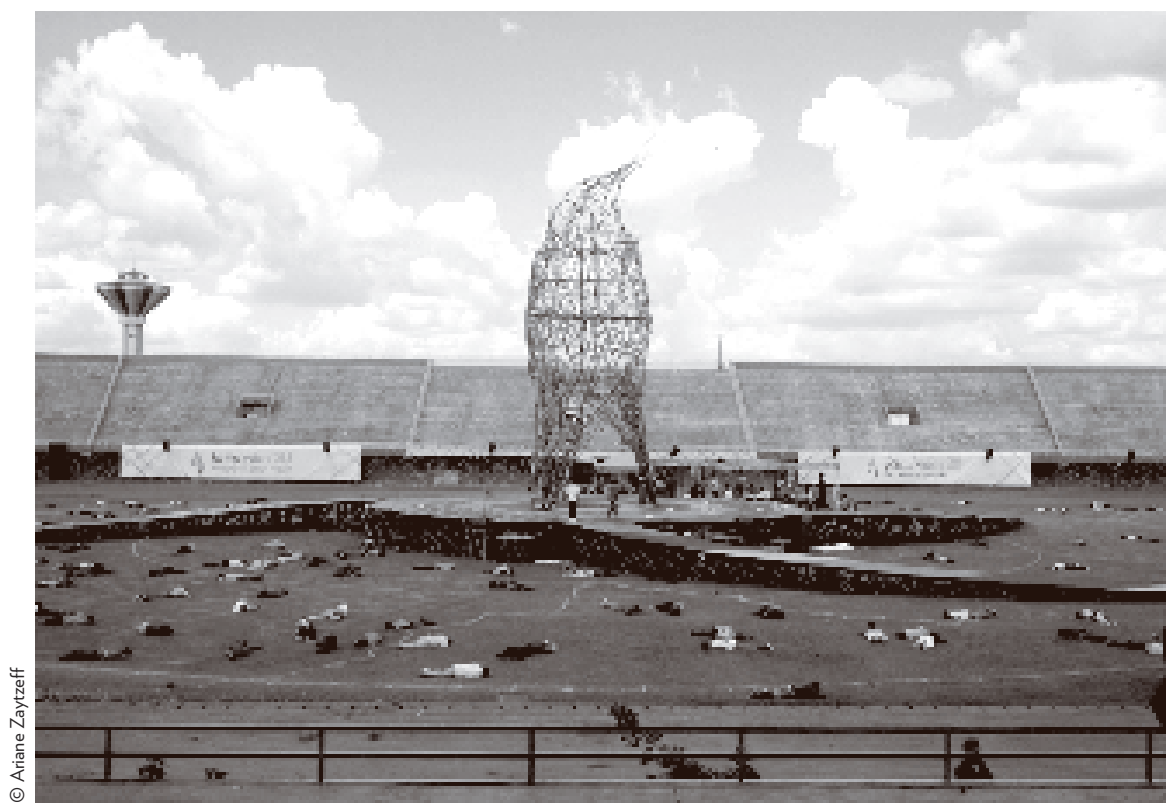




\section{VIOLENCES RADICALES EN SCÈNE}

représentation de la violence et un mode de transmission de sa mémoire, et devenir les moteurs d'une reconstruction culturelle souvent négligée parce qu'immatérielle. Je m’intéresserai donc à des parcours d'artistes rwandais sur la durée pour voir comment chacun appréhende le génocide et l'intègre dans son travail et sa réflexion. En premier lieu, l'étude des productions d'Azeda pour les cérémonies d'ouverture des $10^{\mathrm{e}}$ et $20^{\mathrm{e}}$ commémorations du génocide me permettra d'aborder l'organisation et la construction de la mémoire par le pouvoir politique et de montrer comment la pression du discours officiel limite la créativité de l'artiste. Dans un second temps, j'examinerai la mise en scène de L'Instruction de Peter Weiss par Dorcy Rugamba et Isabelle Gyselinx en 2005 qui propose une approche plus inquisitrice de la mémoire, et la placerai au sein de l'initiative de formation au théâtre contemporain développée par Rugamba pour créer une conversation avec les formes traditionnelles et questionner leur nécessaire réinvention face à la violence du génocide. Enfin, la pièce Ngwino Ubeho, créée en 2009, sera mon point d'entrée dans le parcours artistique d'Odile Gakire Katese, que cette dernière organise autour de la mémoire du génocide tout en engageant un processus de formation artistique qui interroge la place des arts traditionnels et contemporains au sein de la société rwandaise. L'effort affirmé de Rugamba et de Gakire pour le développement des arts au Rwanda fait émerger une réflexion partagée sur la reconstruction culturelle qui tente de répondre aux problèmes de transmission de la mémoire et de recréation du lien social posés par le génocide.

\section{REPRÉSENTER SUR COMMANDE : S'INSÉRER DANS LE CADRE OFFICIEL DE LA MÉMOIRE}

Hope Azeda, créatrice et directrice de la troupe Mashirika, a créé des spectacles pour la cérémonie d'ouverture de la commémoration presque tous les ans depuis dix ans, inscrivant son travail dans le cadre officiel de la mémoire du génocide. C'est en 2004 que le Ministère de la Culture et Aegis Trust, une ONG britannique dédiée à la prévention du génocide, lui demandent pour la première fois de créer une pièce à l’occasion de la dixième commémoration. Azeda explique que le ministère et Aegis ne voulaient pas « que les gens restent assis autour du feu à écouter des témoignages. Ils voulaient quelque chose de nouveau, quelque chose qui donne de l'espoir, qui fasse réfléchir au futur tout en revisitant le passé.» (Azeda, 2012) Le spectacle Rwanda my Hope, joué principalement en anglais avec un peu de kinyarwanda, retrace les événements du génocide à travers l'histoire de plusieurs rescapés, évoque ensuite leurs espoirs pour le futur et finit sur une note positive avec des danses traditionnelles de célébration. Azeda a introduit la danse contemporaine, forme inédite jusque-là, pour exprimer la violence du génocide et la souffrance des rescapés. Elle évoque les fréquentes visites des représentants du ministère, inquiets de l'utilisation d'une forme inconnue, durant les répétitions pour suivre de près la création (Ibid.). Du point de vue du discours sur la mémoire du génocide, la pièce n’ouvre pas de véritable réflexion, mais elle réitère et transmet le discours du gouvernement ainsi que 


\section{DOSSIER}

Créations artistiques au Rwanda après le génocide des Tutsi (suite)

- Répétition de Shadows of Memory, mise en scène d'Azeda.

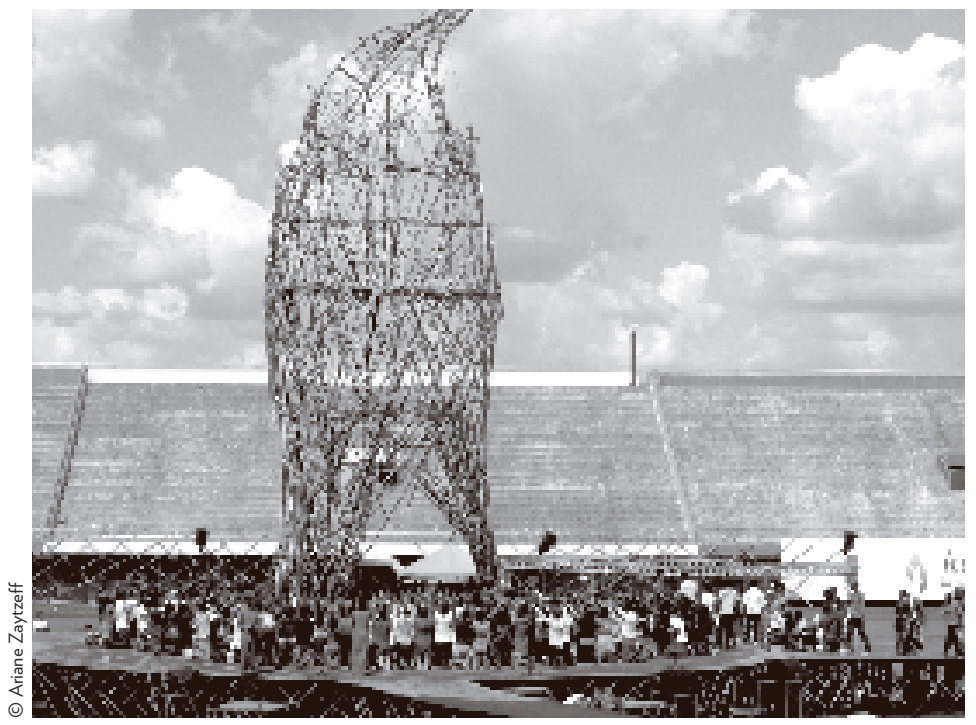

celui d'Aegis Trust, qui a subventionné le spectacle. Le spectacle, par sa composition et le contrôle dont il a été l'objet, est ainsi représentatif de l'organisation et de la construction officielle de la mémoire du génocide.

Dans leur article retraçant l'évolution de la politique mémorielle du gouvernement rwandais, Hélène Dumas et Rémi Korman notent une tendance à « une uniformisation progressive depuis 2008 ", année de la création de la Commission Nationale de Lutte contre le Génocide (CNLG), institution responsable de tout ce qui a trait à l'histoire et à la mémoire du génocide. Ils évoquent notamment « la loi relative aux mémoriaux du génocide qui vise à définir leur contenu, mais aussi leur forme » et qui marque « un encadrement resserré de l'État sur les lieux de mémoire. » (Dumas et Korman, 2011, 24) Le gouvernement fait de ce contenu uniformisé « un récit [...] sanctionné et valorisé par des cadres institutionnels », c'est-à-dire l'histoire, selon la définition de Marita Sturken (Sturken, 1997, 4). C'est donc l'écriture de l'histoire, qui n'a toujours pas trouvé sa place dans les programmes scolaires, qui se joue là, et le contrôle strict du gouvernement s'étend à tout événement officiel de commémoration, qui vient réitérer le discours des mémoriaux. Rwanda my hope ne fait pas exception, et certains des témoignages mis en scène sont pris presque mot pour mot des panneaux du Kigali Genocide Memorial, le mémorial principal et le plus visité, financé et géré par Aegis Trust. La performance reprend les éléments du discours officiel : la présentation d'une unité précoloniale et idéale, l'introduction des différences ethniques et du racisme par les colons, des témoignages de rescapés, des cas de « Justes » qui ont sauvé des Tutsi, et une condamnation de l'inaction de la communauté internationale. C'est une histoire rapide et lacunaire s'écrivant à travers ces éléments qui font partie de l'histoire du Rwanda, mais sont présentés de façon schématique et didactique et laissent peu de place à la réflexion. Comme le remarquent Dumas et Korman dans leur étude du Kigali Genocide Memorial, « les historiens ne sont [...] guère représentés au sein de la direction du centre qui privilégie des profils professionnels centrés sur la défense des droits de l'homme. » (Dumas et Korman, 2011, 19) L'injonction du «plus jamais ça » prend alors le pas sur la réflexion historique et vient soutenir et démontrer l'importance du projet national d'unité et réconciliation.

Dix ans plus tard, pour la vingtième commémoration, Shadows of Memory met en scène, en anglais et en kinyarwanda, le Rwanda avant, pendant, et après le génocide, montrant que 
c'est l'histoire entière du Rwanda qui est définie dans le discours officiel. Dans son entretien avec Philippe Gourevitch, le président Paul Kagame reconnaît en effet que le génocide est un événement essentiel pour comprendre le pays, non seulement son présent et son avenir, mais aussi son passé (Gourevitch, 1996, 164). L’histoire du Rwanda précolonial est représentée par une masse de figurants qui se positionnent en cercle, tournés vers la flamme au centre de la scène, et offrent l'image d'un peuple uni qui regarde dans la même direction.

Cette unité est brisée par l'arrivée des colons qui rompent le cercle et poussent les figurants hors de scène. Les colons échangent ensuite leurs casques pour des bérets bleus et représentent l'ONU quittant la scène alors que le génocide commence. Après les massacres, le FPR entre en scène et relève les victimes, et les figurants sont rejoints par un chœur de deux cents enfants, retrouvant la position circulaire du début. Ce traitement expéditif de l'histoire, qui passe sous silence les deux républiques hutu, est représentatif d'une histoire lue au prisme du génocide qui érige le Rwanda précolonial en symbole d'une unité originelle à retrouver sans prendre en compte les dommages que le génocide a infligé aux valeurs traditionnelles. Pour le psychiatre rwandais Naasson Munyandamutsa, la rupture majeure provoquée par le génocide est celle des liens générationnels, qui assuraient la création d'un sentiment d'appartenance et la cohésion du groupe. Pour se reconstruire, il est nécessaire d'engager une réflexion sur « les valeurs fondatrices de [la] société » qui sont non seulement à retrouver, mais surtout à adapter et à réinventer après la violence qui les a détruites (Munyandamutsa, 2005, 84). Cette réflexion ne se fait qu’à minima dans le discours officiel, qui se positionne plus dans l'affirmation que dans le questionnement de son identité afin de faire avancer la politique d'unité et réconciliation. De plus, le choix de l'anglais comme langue principale de ces spectacles pose la question du public visé qui est autant, si ce n'est plus, le public étranger qui assiste aux commémorations que le public rwandais.

Lors des répétitions d'avril 2014, le service communication de la présidence et Aegis étaient omniprésents et tenaient des réunions avec Azeda et ses collaborateurs après chaque filage. Le contrôle se maintient donc, se durcit même si l'on en croit Dumas et Korman, et plutôt qu'une intervention des arts vivants dans le discours de la mémoire, c'est l'influence du discours officiel sur la pratique d'une artiste qui se dessine. Cette influence semble tendre vers un effacement progressif à la fois des arts et de l'artiste : alors qu'Azeda devait évoquer l'histoire du génocide en cent minutes pour la dixième commémoration, elle a dû pour la vingtième retracer l'histoire du Rwanda en vingt minutes seulement, et elle en a confié la chorégraphie à la Sud-Africaine Penny Jones, spécialiste des chorégraphies de masse. Comme le remarque Connerton dans son étude des cérémonies commémoratives, ces dernières cherchent à établir une stabilité de la mémoire et de l'identité nationales et pour ce faire se basent sur des éléments qu'elles répètent de façon invariable. Cette répétition, à laquelle les performances d'Azeda participent, rend la mémoire présentée peu susceptible à la critique en produisant une habituation des spectateurs (Connerton, 1989, 45 et 102). Certains extraits de Shadows of memory rappellent à nouveau 


\section{DOSSIER}

Créations artistiques au Rwanda après le génocide des Tutsi (suite)

_L'Instruction de

Peter Weiss, mise en scène de Gyselinx et Rugamba. le Kigali Genocide Memorial et d'autres sont des répétitions presque littérales de Rwanda my hope, comme la litanie des nombres qui évoque « un million de morts en cent jours. Dix mille par jour. Quatre cents par heure. Pendant cent jours. » Figées dans le cadre officiel, les performances d'Azeda semblent condamnées à réitérer le discours officiel et à se réitérer elles-mêmes.

\section{DÉPLACER LE DISCOURS, FAIRE JOUER LE THÉÂTRE}

En dehors du cadre officiel, des artistes abordent le théâtre autrement que comme un simple médium à mettre au service d'un discours préétabli, et le travail du Groupov avec Rwanda 94 fait cas d'école sur l'utilisation du théâtre pour explorer l'histoire du génocide et prendre le temps d'interroger ses représentations médiatiques et historiques. L'aventure a regroupé des artistes rwandais qui sont aujourd'hui des figures importantes de la scène culturelle rwandaise, notamment l'auteur, comédien et metteur en scène Dorcy Rugamba. De cette expérience et de la formation qu'il a ensuite suivie au conservatoire de Liège en Belgique vient la conviction que les artistes « ne [sont] pas là pour répondre à la place des gens. [...] C'est ce qui est bien avec les arts vivants, c'est une invitation et les spectateurs font partie du processus. On est ensemble, avec une proposition, et puis ensuite on en discute. » (Rugamba, 2012) Dans cette vision, la performance théâtrale devient ce que Jacques Rancière décrit comme une « troisième chose dont aucun n'est propriétaire, dont aucun ne possède le sens, qui se tient entre eux [artiste et spectateurs], écartant toute transmission à l’identique. » (Rancière, 2008, 21) Cette interaction

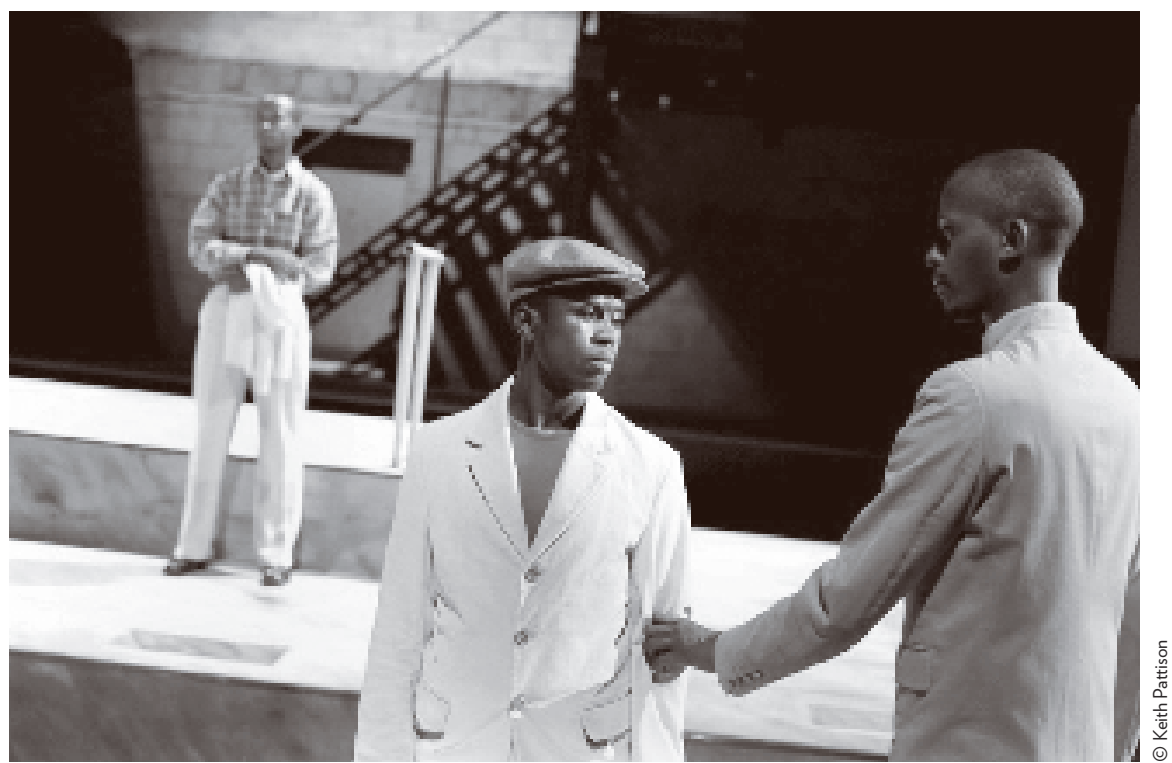




\section{VIOLENCES RADICALES EN SCÈNE}

est propre à l'émancipation des spectateurs, que Rancière définit comme la « vérification de l'égalité des intelligences », et c'est ce que Rugamba souhaite proposer au public rwandais (Ibid., 15-6).

En 2005, il monte avec Isabelle Gyselinx L'Instruction de Peter Weiss, avec des acteurs rwandais. La mise en scène et les costumes, très épurés, ne placent l'action dans aucun contexte historique ou culturel précis et mettent le texte de Weiss, qui porte, lui, les marques de l'Allemagne nazie, à disposition du public de façon à ce qu'il puisse s'en saisir. Les spectateurs peuvent amener le texte vers leurs propres expériences et trouver par exemple dans le drame juridique de Weiss l'écho des gacaca, ces tribunaux qui ont jugé les crimes de génocide à l'échelle locale jusqu’à la fin de leur mandat en 2012. Bien que l'équipe ait effectué un travail de recherche préalable, notamment sur les gacaca, elle ne l'a pas inscrit sur scène et ce sont les spectateurs qui font ce lien, et d'autres encore. Le spectateur est actif au sens où Rancière l'entend, sélectionnant dans le contexte de l'Allemagne nazie ce qui fait sens pour lui, comparant et interprétant pour mettre en perspective le génocide des Tutsi et les institutions et pratiques mises en place pour y faire face. La performance ne prétend ainsi pas délivrer une vérité, mais engager les spectateurs dans la réflexion qu'ouvre l'action jouée sur scène. Le spectacle a surtout été joué en Europe et aux États-Unis, en français d'abord puis en kinyarwanda dans une traduction de Rugamba et Gyselinx. Au Rwanda, il a été joué trois fois, en français au moment de sa création et lors du festival Arts Azimut, organisé par Gakire en 2008, puis en kinyarwanda en 2012. Rugamba remarque qu'en kinyarwanda le lien avec les gacaca se faisait encore plus naturellement pour les spectateurs rwandais (Rugamba, 2012). Cependant, L'Instruction n'a selon lui toujours pas rencontré le public qu'il espérait au Rwanda, c'est-à-dire un public populaire, pas uniquement composé de professeurs et de lettrés. Au-delà de la langue, c'est l'espace qui pose problème, d'autant plus depuis la fermeture du centre d'art Ishyo, dirigé par Carole Karemera, en 2013. Les salles sont peu accessibles : l'auditorium de l'université du Rwanda à Butare, quand il est disponible, attire surtout les étudiants et professeurs, la salle du Rwanda Revenue à Kigali, située dans les bureaux du Ministère des Finances, est peu connue du public, et les espaces indépendants ne sont pas équipés pour accueillir des spectacles.

La réflexion que propose L'Instruction nécessite donc d'être diffusée plus largement, mais le travail de Rugamba dépasse évidemment ce seul spectacle, qu'il a créé au terme d'une série d'ateliers, baptisés Urwintore (la scène, le lieu des artistes). Organisés de 2001 à 2005 avec l'aide de professeurs du conservatoire de Liège, dont Gyselinx, leur but était de former des acteurs rwandais à différentes techniques de jeu. En effet, le Rwanda n’a pas de tradition théâtrale, et même si certains éléments de la culture traditionnelle sont très théâtraux, « le théâtre contemporain [...] nécessitait de passer par une longue phase d'apprentissage. De poser d'ailleurs des questions. » (Rugamba, 2012) Rugamba interroge donc la nature du médiumthéâtre et son propre choix de passer par cette forme étrangère : pourquoi s'initier à ces techniques théâtrales? Que peuvent-elles apporter aux problématiques locales? 


\section{DOSSIER}

Créations artistiques au Rwanda après le génocide des Tutsi (suite)

Comment les adapter à la culture rwandaise? Il s'agit alors d'interroger, réinventer et transmettre la mémoire culturelle, que Sturken définit comme une mémoire collective qui se crée hors du discours historique officiel, mais reste cependant porteuse d'identité et signification culturelles (Sturken, 1997, 1-2). Bien que basé à Bruxelles depuis 1994, Rugamba séjourne régulièrement au Rwanda et s’investit dans le déve-

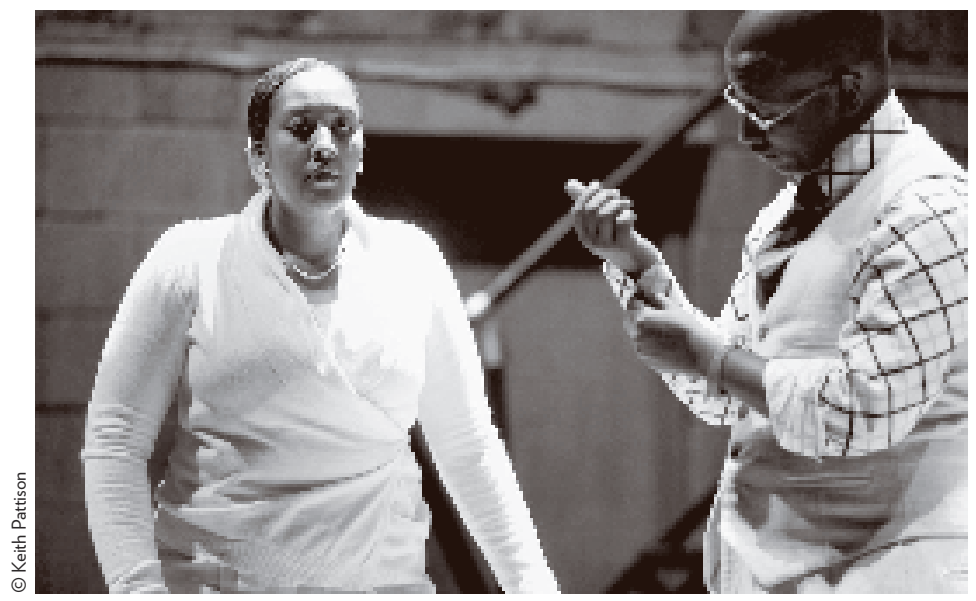

loppement du secteur culturel, car il est pour lui important «que nous Rwandais nous fassions quelque chose qui vienne d'ici. [...] Que ce soit aussi une initiative qui soit la nôtre. » (Rugamba, 2012)

L'introduction de la forme théâtrale produit ce que Diana Taylor appelle une «transculturation », c'est-à-dire « le processus de transformation qu'opère une société lorsqu'elle acquiert un élément culturel étranger [...] et la fusion de l'indigène et de l'étranger pour créer un produit culturel nouveau et original. » (Taylor, 2003, 104) Dans le cas du Rwanda, la nouveauté qu'apporte le théâtre vient peut-être répondre à la difficulté de dire et de représenter un

_L'Instruction de Peter Weiss, mise en scène de Gyselinx et Rugamba. événement qui, dans son exécution comme sa préparation, a dévasté la culture rwandaise. Pour l'historienne et psychologue sociale Assumpta Mugiraneza, «le génocide s'est fait parce que nous avions perdu notre culture, nos racines, nos références. » (Mugiraneza, 2012) En effet, le système politique qui a précédé et mené au génocide imposait une lourde censure aux arts, provoquant ce que l'auteur et metteur en scène rwandais Kalisa Rugano appelle une « béance culturelle » (Rugano, 2012). Bien qu'aujourd'hui le travail de reconstruction du Rwanda inclue une réactivation de la culture traditionnelle, celle-ci se fait généralement sans questionner l'évolution nécessaire des pratiques culturelles afin de les replacer au centre du quotidien. Sans cette évolution, comme le remarque Rugamba, «la tradition se trouve très démunie pour traduire ça [le génocide]. » (Rugamba, 2012) On touche à la fois à la mémoire (donc construction) qu'un peuple a de sa culture et à la mémoire que porte cette culture, ici la mémoire d'une violence qui se manifeste par des ruptures dans la transmission orale. Pour Munyandamutsa, le génocide a détruitle «dispositiffamilial et communautaire » qui était le «vecteur de la culture » et il a donc mis en danger sa transmission (Munyandamutsa, 2005, 84). Si une culture peut se souvenir, comme le suggère Sturken, elle a besoin des individus et repose sur leurs interactions afin de créer sa mémoire et sa signification (Sturken, 1997, 1). L'intervention du théâtre, comme un espace d'engagement des artistes et des spectateurs où repenser les pratiques et réalités rwandaises, vient nourrir la culture en vue de l'aider non seulement à appréhender la mémoire du génocide, mais aussi à se reconstruire elle-même. 
Lorsqu'elle crée Ngwino Ubeho (Viens et sois en vie) en 2009, Odile Gakire Katese ajoute à la forme du théâtre celle tout aussi nouvelle de la danse contemporaine avec la chorégraphe française Flora Théfaine, et fait appel à une tradition musicale étrangère avec le musicien burkinabé Alif Naaba. Cette transculturation multiple est mise en œuvre sur scène et en dehors par l'organisation d'ateliers de formation pour les danseurs et chanteurs rwandais au Centre Universitaire des Arts de Butare. La danse traditionnelle intervient par moments et dialogue avec la danse contemporaine, et la musique de Naaba est interprétée par des musiciens et certains instruments rwandais. De cette alliance naît une performance qui conjure le passé au sein d'un présent marqué par l'absence. Dans le discours de la commémoration, on évoque la violence du génocide dans le passé mais on n'explore guère la violence du présent, faite de solitude et d'absence pour les rescapés en particulier. Le discours officiel renvoie l'expression des mémoires individuelles dans les marges de la scène publique, et c'est dans ces marges que travaille Gakire, avec une approche dynamique de la mémoire. Il ne s'agit pas de s’aligner sur une mémoire préétablie, mais de la provoquer pour aller chercher plus loin :

Aujourd'hui, je convoque l'histoire

Pour lui rafraîchir la mémoire.

Au besoin, je la fouetterai

Pour lui faire avouer

Ce qu'elle n'a pas encore dit (Gakire, 2009, 4).

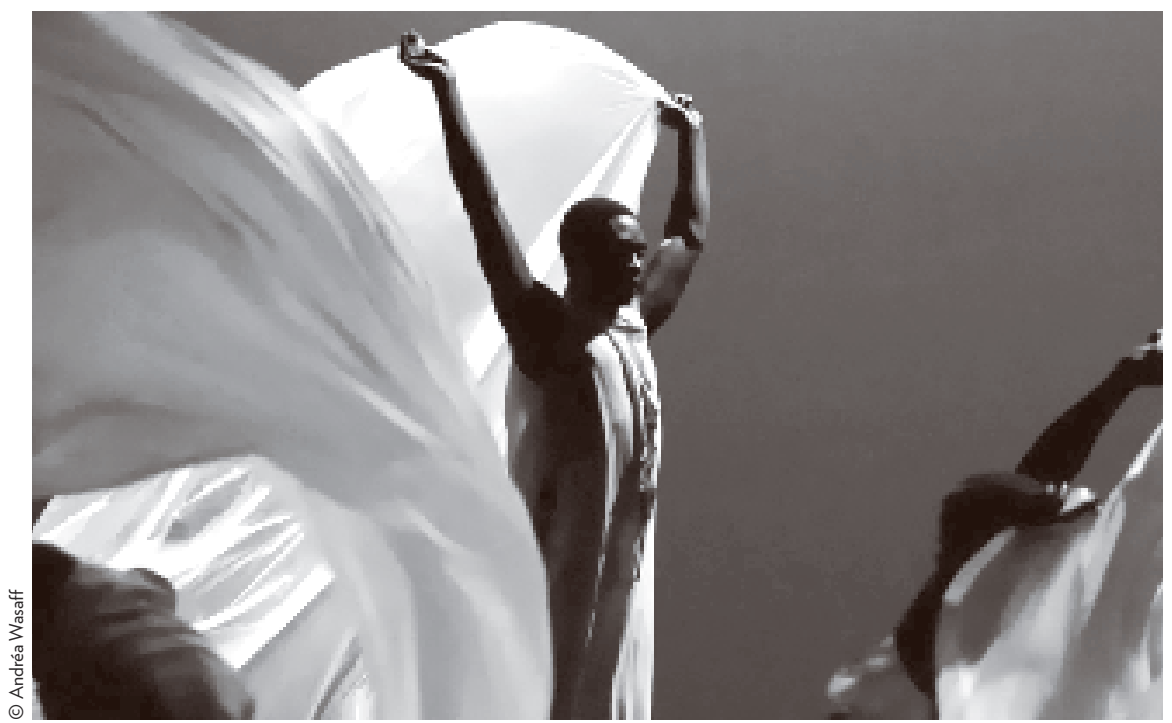

_ Ngwino Ubeho, texte et mise en scène de Gakire. 


\section{DOSSIER}

Créations artistiques au Rwanda après le génocide des Tutsi (suite)

Cette approche est représentative du travail de Gakire, qui ne se satisfait pas de l'histoire telle qu'elle est écrite et envisage le travail de mémoire comme une lutte de longue haleine pour dire plus et autrement. En cherchant à dire ce qui est absent du discours officiel, ce sont les absents eux-mêmes qu'elle appelle afin de les réintégrer dans la reconstruction du présent. Avec la danse contemporaine, elle donne corps aux morts et leur offre une place et une présence sur scène.

Son texte aussi tisse des liens avec les victimes du génocide en proposant, à l'exception d'une scène dialoguée, une adresse poétique à un « tu » disparu qui peut être l'enfant, le parent, l'amant, l'ami. Cette adresse propose une mémoire personnelle et s'offre au spectateur pour qu'il puisse l'habiter et parler à son tour à

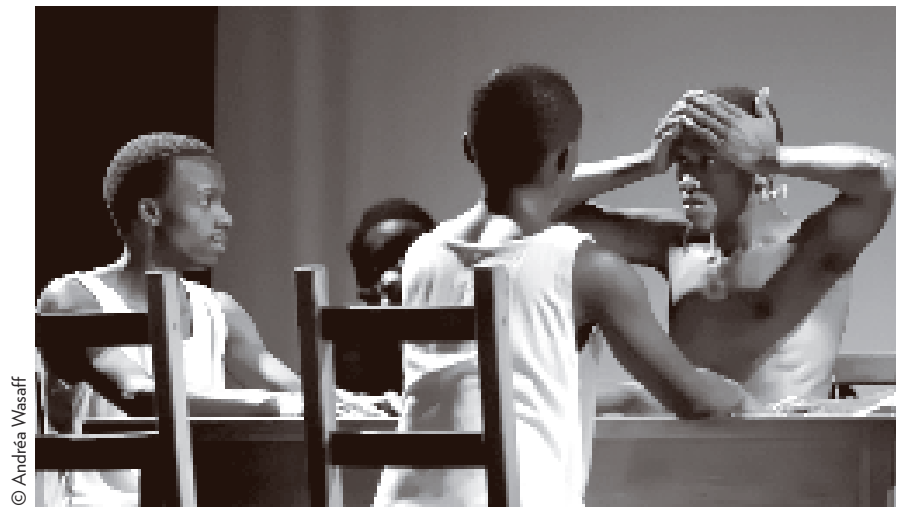

_ Ngwino Ubeho, texte et mise en scène de Gakire. ses morts, répondant ainsi au besoin crucial identifié par Munyandamutsa de «se structurer autour de la mémoire des siens disparus, qui ont constitué le fondement de sa vie » afin de pouvoir construire un récit personnel « qui consolide la refondation identitaire. » (Munyandamutsa, 2005, 87) En mettant en scène un lien verbal et visuel avec les morts, Gakire donne à voir et à entendre comment les vivants peuvent, par le souvenir, redonner vie aux morts dans leur quotidien. Dans une des scènes, un rescapé dîne à une table en présence de ses fantômes. Il parle seul, rit, et on entend en fond des bruits enregistrés qui recréent l'atmosphère des dîners où la famille était présente. Un danseur/fantôme monte sur la table et danse dans le style traditionnel des Intore qui évoque le passé disparu, mais le rescapé ne voit pas les fantômes et ceux-ci ne lui répondent pas.

Les morts trouvent ainsi une place sur scène grâce à leur relation avec les vivants, à travers un lien émotionnel notamment: Gakire évoque les yeux, la peau, le cœur, la peur, la colère de la victime, qui deviennent ce que les vivants portent et font exister dans leurs mémoires et leurs paroles. Pour construire cette mémoire intime, il faut se détacher de la mémoire officielle des mémoriaux et des événements de commémoration :

J'extrais ton nom des sites mémoriaux

Et je rappelle au souvenir du monde

Qu'hier encore tu étais

Qu'aujourd'hui, tu n'es plus

Mais que demain tu seras (Gakire, 2009, 4).

La volonté de s' « extraire » des monuments et discours qui contiennent la mémoire du génocide vise à la faire déborder et dépasser les frontières qu’on lui 


\section{VIOLENCES RADICALES EN SCÈNE}

impose. Malgré le constat de l'absence au présent - «tu n’es plus » - la mémoire s'inscrit dans le futur avec l'engagement de faire exister le mort demain - « tu seras ». Le rapport au passé est donc en constant mouvement et ce dernier est réactivé au quotidien par la mémoire des vivants.

Ce rapport dynamique se ressent dans le travail de Gakire, qui remet en jeu son propre rapport à la mémoire du génocide au fil de ses créations. Convaincue que « dans un pays comme le Rwanda, on ne peut pas ne pas s'arrêter pour faire un travail sur la mémoire », elle a choisi de faire un projet sur le génocide tous les cinq ans (Gakire, 2012). Avec Ngwino Ubeho, elle écrit une réponse aux spectateurs d'Iryo Nabonye (Ce que j'ai vu), créé en 2004 avec Jean-Claude Uwilingiyimana et Aimable Twahirwa, qui avait provoqué des crises émotionnelles dans le public. Face à ces réactions, Gakire s'oriente après ce premier spectacle vers la quête d'une mémoire apaisée, qui passe pour elle par la recréation d'un dialogue avec les morts afin d'esquisser une mémoire plus vivante. Pourtant Ngwino Ubeho, en faisant ressentir l'absence de façon particulièrement poignante, a malgré tout provoqué des pleurs et a de ce fait amené Gakire à questionner son envie d'éviter les larmes. Ainsi, malgré son intention et ses efforts, elle a appris avec ses créations successives à «apprivoiser » ses attentes, comme elle aime le dire, et à diversifier son approche. En mettant en œuvre sur scène ce qu'elle souhaite apporter à la mémoire du génocide, les performances et discussions avec le public lui donnent en retour des éléments de réponse et de nouvelles questions qui nourrissent sa compréhension du génocide. Le théâtre devient alors un outil d'investigation du génocide, de ses effets, et de sa place dans le Rwanda d'aujourd'hui, se nourrissant de son interaction avec le public pour prendre le pouls de la situation actuelle et ajuster son intervention aux interrogations qui surgissent. Comme pour L'Instruction, le public rencontré est cependant resté limité. Gakire a écrit le texte de Ngwino Ubeho en français, et bien que la plus grande partie de la pièce, la musique et la danse soit accessible à tous, le choix du français occulte une partie aux spectateurs qui ne parlent pas cette langue. De plus, les besoins techniques du spectacle ont fait qu'il n’a été joué qu’au grand auditorium de l'université nationale à Butare, seule salle véritablement équipée, puis à Nantes en France.

Les problèmes d'accessibilités de L'Instruction et de Ngwino Ubeho, du fait de la langue et de l'espace, limitent leur diffusion, mais n'enlèvent rien au potentiel de la vision et de la pensée qu'ils offrent. Le travail de Gakire et de Rugamba s’insère dans des réflexions personnelles sur la construction de la mémoire du génocide et l'importance de la culture dans la reconstruction du pays. Les deux spectacles se sont accompagnés de formations en théâtre contemporain et en danse contemporaine qui ont dépassé le cadre de la création : les ateliers Urwintore ont duré de 2001 à 2005, et Gakire, en tant que directrice adjointe du Centre Universitaire des Arts à Butare de 2003 à 2011, a offert toute une série de formations, en théâtre et en danse particulièrement. Ces formations ne concernent pas uniquement les formes contemporaines, et Gakire a créé en 2004 la troupe Ingoma Nshya (Nouveau Tambour) composée uniquement de femmes qui ont été formées au tambour 
Créations artistiques au Rwanda après le génocide des Tutsi (suite) traditionnel, jusque-là réservé aux hommes. En s'appropriant une forme qui leur était interdite, les femmes l'ont à la fois réactivée, questionnée et changée. Gakire et Rugamba ne sont donc pas seulement metteurs en scène, mais s'efforcent aussi d'organiser et d'aider à produire d'autres artistes. Depuis 2012, Rugamba développe le projet Rwanda Arts Initiative pour proposer des formations qui touchent à tous les métiers artistiques, avec une attention particulière aux aspects technique et administratif de la création, qui manquent cruellement au Rwanda. En formant des producteurs et des administrateurs de projet, il s'agit de créer les conditions et le soutien nécessaires pour permettre aux artistes de se développer et de diffuser leur travail plus largement, améliorant ainsi leur visibilité et leur intégration dans la société rwandaise et à l'étranger.

Ces projets plus larges n'évoquent pas directement la mémoire du génocide, mais ne sont néanmoins pas sans lien avec celle-ci. Avec un ministère des sports et de la culture qui propose peu de soutien aux arts et qui tend à confondre culture avec tourisme, ce sont des initiatives personnelles comme celles de Gakire et Rugamba, ainsi que d'autres artistes et entrepreneurs rwandais, qui développent une vision dynamique de la culture et engagent une réflexion sur son évolution face à la violence extrême qui l’a mise à mal.

\section{BIBLIOGRAPHIE}

- Azeda, Hope (24 mai 2012) : «The role of the arts in reconciliation », table ronde, Kigali, Rwanda.

- Connerton, Paul (1989) : How societies remember, New York, Cambridge University Press.

- Dumas, Hélène et Korman, Rémi (2011) : « Espaces de la mémoire du génocide des Tutsis au Rwanda. Mémoriaux et lieux de mémoire », Afrique contemporaine, $n^{\circ} 238$, p. 11-27.

- Gakire, Odile (2009) : Ngwino Ubeho ou la pluie et les larmes, manuscrit non publié, Butare, Rwanda.

- Gourevitch, Philip (1996) : «After genocide: a conversation with Paul Kagame », Transition, n 72, p. $162-94$.

- Mugiraneza, Assumpta (21 novembre 2012) : Entretien avec l’auteur, Kigali.

- Munyandamutsa, Naasson (2005) : «Renouer avec les liens générationnels », in Jean Furtos et Christian Laval (dir.), La santé mentale en actes, ERES «Études, recherches, actions en santé mentale en Europe », p. 83-89.

- Rancière, Jacques (2008) : Le spectateur émancipé, Paris, La Fabrique.

- Rugamba, Dorcy (17 décembre 2012) : Entretien avec l’auteur, Kigali.

- Rugano, Kalisa (17 décembre 2012) : Entretien avec l'auteur, Kigali.

- Sturken, Marita (1997) : Tangled memories: the Vietnam war, the AIDS epidemic, and the politics of remembering, Berkeley, University of California Press.

- Taylor, Diana (2003) : The archive and the repertoire: performing cultural memory in the Americas, Durham, Duke University Press. 